\title{
Starlike Functions Related to the Bell Numbers
}

\author{
Nak Eun Cho ${ }^{1, *}$, Sushil Kumar ${ }^{2}$, Virendra Kumar ${ }^{3}$ (D), V. Ravichandran ${ }^{4}$ (D) \\ and H. M. Srivastava $5,6, *$ (D) \\ 1 Department of Applied Mathematics, Pukyong National University, Busan 48513, Korea \\ 2 Bharati Vidyapeeth's College of Engineering, Delhi 110063, India; sushilkumar16n@gmail.com \\ 3 Department of Mathematics, Ramanujan College, University of Delhi, Kalkaji, New Delhi 110019, India; \\ vktmaths@yahoo.in \\ 4 Department of Mathematics, National Institute of Technology, Tiruchirappalli, Tamil Nadu 620015, India; \\ vravi68@gmail.com or ravic@nitt.edu \\ 5 Department of Mathematics and Statistics, University of Victoria, Victoria, BC V8W 3R4, Canada \\ 6 Department of Medical Research, China Medical University Hospital, China Medical University, \\ Taichung 40402, Taiwan \\ * Correspondence: necho@pknu.ac.kr (N.E.C.); harimsri@math.uvic.ca (H.M.S.)
}

Received:19 January 2019; Accepted: 12 February 2019; Published: 13 February 2019

\begin{abstract}
The present paper aims to establish the first order differential subordination relations between functions with a positive real part and starlike functions related to the Bell numbers. In addition, several sharp radii estimates for functions in the class of starlike functions associated with the Bell numbers are determined.
\end{abstract}

Keywords: differential subordination; starlike functions; Bell numbers; radius estimate

MSC: 30C45; 30C55; 30C80

\section{Introduction}

Let $\mathcal{A}$ be a class of analytic functions $f$ in the open unit disk $\mathbb{D}:=\{z \in \mathbb{C}:|z|<1\}$ and normalized by the conditions $f(0)=0$ and $f^{\prime}(0)=1$. Suppose $\mathcal{S}$ is a subclass of $\mathcal{A}$ consisting of univalent functions. An analytic function $f$ is subordinate to $g$, written as $f \prec g$, if there exists an analytic function $w: \mathbb{D} \rightarrow \mathbb{D}$ with $|w(z)| \leq|z|$ such that $f(z)=g(w(z))(z \in \mathbb{D})$. Moreover, if $g$ is univalent in $\mathbb{D}$, then the equivalent conditions for subordination can be written as $f(0)=g(0)$ and $f(\mathbb{D}) \subseteq g(\mathbb{D})$. By imposing some geometric and analytic conditions over the functions in the class $\mathcal{S}$, many authors considered several subclasses of $\mathcal{S}$. Various subclasses of starlike and convex functions were studied in the literature, and they can be unified by considering an analytic univalent function $\varphi$ with a positive real part in $\mathbb{D}$, symmetric about the real axis and starlike with respect to $\varphi(0)=1$, and $\varphi^{\prime}(0)>0$. Ma and Minda [1] studied the class

$$
\mathcal{S}^{*}(\varphi):=\left\{f \in \mathcal{A}: \frac{z f^{\prime}(z)}{f(z)} \prec \varphi(z)\right\} .
$$

The class $\mathcal{S}^{*}(\varphi)$ for various choice of the domain $\varphi(\mathbb{D})$ was considered in recent years. The class $\mathcal{S}^{*}[A, B]:=\mathcal{S}^{*}((1+A z) /(1+B z))(-1 \leq B<A \leq 1)$ was introduced by Janowski [2]. For $0 \leq \alpha \leq 1$, the class $\mathcal{S}^{*}(\alpha):=\mathcal{S}^{*}[1-2 \alpha,-1]$ is the class of starlike functions of order $\alpha$. Uralegaddi et al. [3] defined the class

$$
\mathcal{M}(\beta):=\left\{f \in \mathcal{A}: \operatorname{Re}\left(\frac{z f^{\prime}(z)}{f(z)}\right)<\beta(\beta>1)\right\}=\mathcal{S}^{*}\left(\frac{1+(1-2 \beta) z}{1-z}\right) .
$$


Several authors considered various special cases of the class of Janowski starlike functions by considering some specific functions, namely $\varphi_{q}(z):=z+\sqrt{1+z^{2}}, \varphi_{0}(z):=1+$ $(z / k)((k+z) /(k-z))(k=\sqrt{2}+1), \varphi_{s}(z):=1+\sin z$, and $G_{\alpha}(z):=1+z /\left(1-\alpha z^{2}\right)$. Some of those classes are: $\mathcal{S}_{L}^{*}:=\mathcal{S}^{*}(\sqrt{1+z})$ [4], $\mathcal{S}_{q}^{*}:=\mathcal{S}^{*}\left(\varphi_{q}(z)\right)$ [5], $\mathcal{S}_{e}^{*}=\mathcal{S}^{*}\left(e^{z}\right)$ [6], $\mathcal{S}_{R}^{*}=\mathcal{S}^{*}\left(\varphi_{0}\right)$ [7], $\left.\mathcal{S}_{s}^{*}=\mathcal{S}^{*}\left(\varphi_{s}\right)[8]\right), \mathcal{B S}^{*}(\alpha):=\mathcal{S}^{*}\left(G_{\alpha}(z)\right), 0 \leq \alpha<1[9,10]$. For a brief survey on these classes, readers may refer to $[11,12]$.

It should be noted that the special cases of $\varphi$, mentioned above, are univalent in the unit disk. In 2011, Dziok et al. $[13,14]$ considered $\varphi$ to be a non-univalent function associated with the Fibonacci numbers, defined by

$$
\tilde{p}(z):=\varphi(z)=\frac{1+\tau^{2} z^{2}}{1-\tau z-\tau^{2} z^{2}}, \quad \tau:=(1-\sqrt{5}) / 2
$$

which maps the unit disk $\mathbb{D}$ on to a shell-like domain in the right-half plane. Further, they defined the class $\mathcal{S}_{F}^{*}:=\left\{f \in \mathcal{A}: z f^{\prime}(z) / f(z) \prec \tilde{p}(z)\right\}$. The functions $f \in \mathcal{S}_{F}^{*}$ are starlike of order $\sqrt{5} / 10$.

Motivated by the above defined classes, we consider a function associated with the Bell Numbers. For a fixed non-negative integer $n$, the Bell numbers $B_{n}$ count the possible disjoint partitions of a set with $n$ elements into non-empty subsets or, equivalently, the number of equivalence relations on it. The Bell numbers $B_{n}$ satisfy a recurrence relation involving binomial coefficients $B_{n+1}=\sum_{k=0}^{n}\left(\begin{array}{c}n \\ k\end{array}\right) B_{k}$. Clearly $B_{0}=B_{1}=1, B_{2}=2, B_{3}=5, B_{4}=15, B_{5}=52$, and $B_{6}=203$. For more details, see [15-21]. Kumar et al. [22] considered the function

$$
Q(z):=e^{e^{z}-1}=\sum_{n=0}^{\infty} B_{n} \frac{z^{n}}{n !}=1+z+z^{2}+\frac{5}{6} z^{3}+\frac{5}{8} z^{4}+\cdots(z \in \mathbb{D})
$$

which is starlike with respect to 1 and it's coefficients generate the Bell numbers. Kumar et al. [22] defined the class $\mathcal{S}_{B}^{*}$ by $\mathcal{S}_{B}^{*}:=\mathcal{S}^{*}(Q)$. From [1], note that the function $f \in \mathcal{S}_{B}^{*}$ if and only if there exists an analytic function $q$, satisfying $q(z) \prec Q(z)(z \in \mathbb{D})$, such that

$$
f(z)=I(q(z))=z \exp \left(\int_{0}^{z} \frac{q(t)-1}{t} d t\right) .
$$

The above representation shows that the functions in the class $\mathcal{S}_{B}^{*}$ can be seen as an integral transform $I(q(z))$ of the function $q$ with $f(0)=0$ and $f^{\prime}(0)=1$. The reader may refer to the paper [23] and the references cited therein for integral transform related works. The authors in [22] determined sharp coefficient bounds on the six initial coefficients, Hankel determinant, and on the first three consecutive higher order Schwarzian derivatives for functions in the class $\mathcal{S}_{B}^{*}$.

Let $\mathcal{P}$ be the class of analytic functions $p: \mathbb{D} \rightarrow \mathbb{C}$ with $p(0)=1$ and $\operatorname{Re} p(z)>0(z \in \mathbb{D})$. In 1989, Nunokawa et al. [24] showed that if $1+z p^{\prime}(z) \prec 1+z$, then $p(z) \prec 1+z$. In 2007, Ali et al. [25] computed the condition on $\beta$, in each case, for which

$$
1+\frac{\beta z p^{\prime}(z)}{p^{j}(z)} \prec \frac{1+D z}{1+E z}(j=0,1,2) \text { implies } p(z) \prec \frac{1+A z}{1+B z},
$$

$A, B, C, D, E, F \in[-1,1]$. Further, Ali et al. [26] determined some sufficient conditions for normalized analytic functions to lemniscate starlike functions. Recently, Kumar and Ravichandran [27] obtained sufficient conditions for first order differential subordinations so that the corresponding analytic function belongs to the class $\mathcal{P}$. In 2016, Tuneski [28] gave a criteria for analytic functions to be Janowski starlike. For more details, see [11,29-33].

Motivated by above works, in Section 2, using the theory of differential subordination developed by Miller and Mocanu, a sharp bound on parameter $\beta$ is determined in each case so that $p(z) \prec Q(z)$, whenever $1+\beta z p^{\prime}(z) / p^{j}(z)(j=0,1,2)$ is subordinate to the function $\varphi_{0}(z)$ or $\sqrt{1+z}$ or $G_{\alpha}(z)$ or $(1+A z) /(1+B z)$ or $\varphi_{s}(z)$ or $\varphi_{q}(z)$. Further, various sufficient conditions are obtained for $f \in \mathcal{A}$ to be 
in the class $\mathcal{S}_{B}^{*}$ as an application of these subordination results. In Section 3, $\mathcal{S}_{B}^{*}$-radius for the class of Janowski starlike functions and some other well-known classes of analytic functions are investigated.

\section{Differential Subordinations}

Theorem 1 provides estimate on $\beta$ so that $p(z) \prec Q(z)$ holds, whenever $1+\beta z p^{\prime}(z) \prec \varphi_{0}(z)$ or $\varphi_{s}(z)$ or $\sqrt{1+z}$ or $G_{\alpha}(z)$ or $(1+A z) /(1+B z)$ or $\varphi_{s}(z)$ or $\varphi_{q}(z)$ or $e^{z}$.

To prove our main results, we need the following lemma due to Miller and Mocanu:

Lemma 1. ([32] Theorem $3.4 \mathrm{~h}, \mathrm{p} .132)$ Let $q$ be analytic in $\mathbb{D}$ and let $\psi$ and $v$ be analytic in a domain $U$ containing $q(\mathbb{D})$ with $\psi(w) \neq 0$ when $w \in q(\mathbb{D})$. Set

$$
\mathcal{Q}(z):=z q^{\prime}(z) \psi(q(z)) \text { and } h(z):=v(q(z))+\mathcal{Q}(z) .
$$

\section{Suppose that}

(i) either $h$ is convex, or $\mathcal{Q}$ is starlike univalent in $\mathbb{D}$ and

(ii) $\operatorname{Re}\left(\frac{z h^{\prime}(z)}{\mathcal{Q}(z)}\right)>0$ for $z \in \mathbb{D}$.

If $p$ is analytic in $\mathbb{D}$, with $p(0)=q(0), p(\mathbb{D}) \subseteq U$ and

$$
v(p(z))+z p^{\prime}(z) \psi(p(z)) \prec v(q(z))+z q^{\prime}(z) \psi(q(z)),
$$

then $p \prec q$, and $q$ is most dominant.

Theorem 1. Let $l(e)=\left(1-e^{(1-e) / e}\right)^{-1}, 0<\alpha<1,0<B<A<1$, and $p$ be an analytic function defined in $\mathbb{D}$ with $p(0)=1$.

Set

$$
\mathrm{Y}_{\beta}(z, p(z))=1+\beta z p^{\prime}(z) .
$$

Then, the following are sufficient for $p(z) \prec Q(z)$.

(a) $\mathrm{Y}_{\beta}(z, p(z)) \prec \varphi_{0}(z)$ for $\beta \geq l(e)(1-\sqrt{2}+\log 2) \approx 0.59533$.

(b) $\mathrm{Y}_{\beta}(z, p(z)) \prec \sqrt{1+z}$ for $\beta \geq l(e)(2(1-\log 2)) \approx 1.30984$.

(c) $Y_{\beta}(z, p(z)) \prec G_{\alpha}(z)$ for $\beta \geq l(e) \frac{1}{2 \sqrt{\alpha}} \log \frac{1+\sqrt{\alpha}}{1-\sqrt{\alpha}}$.

(d) $\mathrm{Y}_{\beta}(z, p(z)) \prec \frac{1+A z}{1+B z}$ for $\beta \geq l(e) \frac{A-B}{B} \log (1-B)^{-1}$.

(e) $\mathrm{Y}_{\beta}(z, p(z)) \prec \varphi_{s}(z)$ for $\beta \geq l(e) \sum_{n=0}^{\infty} \frac{(-1)^{n}}{(2 n+1) !(2 n+1)} \approx 2.01905$.

(f) $\mathrm{Y}_{\beta}(z, p(z)) \prec \varphi_{q}(z)$ for $\beta \geq l(e)(2-\sqrt{2}-\log 2+\log (1+\sqrt{2})) \approx 1.65198$.

(g) $\mathrm{Y}_{\beta}(z, p(z)) \prec e^{z}$ for $\beta \geq l(e) \sum_{n=0}^{\infty} \frac{(-1)^{n-1}}{n ! n} \approx 0.785166$.

The lower bound on $\beta$ in each case is sharp.

Proof. Let the functions $v$ and $\psi$ be defined by $v(w)=1$ and $\psi(w)=\beta$.

(a) Define the function $q_{\beta}: \overline{\mathbb{D}} \rightarrow \mathbb{C}$ by

$$
q_{\beta}(z)=1-\frac{1}{\beta k}\left(z+2 k \log \left(1-\frac{z}{k}\right)\right)
$$

is a solution of the differential equation $\beta z q^{\prime}(z)=\varphi_{0}(z)-1$ and is analytic in $\mathbb{D}$. Now consider the function

$$
\mathcal{Q}(z)=z q_{\beta}^{\prime}(z) \psi\left(q_{\beta}(z)\right)=\varphi_{0}(z)-1=\frac{k+z-2 k^{2}}{k-z} .
$$

It can be easily seen that $\mathcal{Q}$ is starlike in $\mathbb{D}$ and the function $h$ is defined by

$$
h(z):=v(q(z))+\mathcal{Q}(z)=1+\mathcal{Q}(z)
$$


satisfies the following inequality

$$
\operatorname{Re}\left(\frac{z h^{\prime}(z)}{\mathcal{Q}(z)}\right)=\operatorname{Re}\left(\frac{z \mathcal{Q}^{\prime}(z)}{\mathcal{Q}(z)}\right)>0(z \in \mathbb{D})
$$

Therefore, from Lemma 1, we conclude that

$$
1+\beta z p^{\prime}(z) \prec 1+\beta z q_{\beta}^{\prime}(z) \text { implies } p \prec q_{\beta} \text {. }
$$

Now the subordination $p \prec Q$ holds if subordination $q_{\beta} \prec Q$. Thus, the subordination $q_{\beta} \prec Q$ holds if the inequalities

$$
Q(-1) \leq q_{\beta}(-1) \leq q_{\beta}(1) \leq Q(1)
$$

hold and these yield a necessary condition for subordination $p \prec Q$ to hold. In view of the graph of the respective function, the necessary condition is also sufficient condition. The inequalities $q_{\beta}(-1) \geq Q(-1)$ and $q_{\beta}(1) \leq Q(1)$ yield $\beta \geq \beta_{1}$ and $\beta \geq \beta_{2}$, where

$$
\beta_{1}=\frac{1-\sqrt{2}+\log 2}{1-e^{(1-e) / e}} \text { and } \beta_{2}=\frac{1-\sqrt{2}-2 \log (2-\sqrt{2})}{e^{(e-1) / e}-1} .
$$

Now the subordination $q_{\beta} \prec Q$ holds if $\beta \geq \max \left\{\beta_{1}, \beta_{2}\right\}=\beta_{1}$.

(b) The function

$$
q_{\beta}(z)=\frac{\beta+2(\sqrt{1+z}-\log (1+\sqrt{1+z})+\log 2-1)}{\beta}
$$

is an analytic solution of the first order differential equation $\beta z q^{\prime}(z)=\sqrt{1+z}-1$ in $\mathbb{D}$. The function $\mathcal{Q}$ defined by $\mathcal{Q}(z)=z q_{\beta}^{\prime}(z) \psi\left(q_{\beta}(z)\right)=\sqrt{1+z}-1$ is starlike in $\mathbb{D}$ and the function $h(z):=$ $v(q(z))+\mathcal{Q}(z)$ satisfies $\operatorname{Re}\left(z h^{\prime}(z) / \mathcal{Q}(z)\right)=\operatorname{Re}\left(z \mathcal{Q}^{\prime}(z) / \mathcal{Q}(z)\right)>0, z \in \mathbb{D}$. Therefore, in view of the subordination relation 1 , the required subordination $p \prec Q$ holds if subordination $q_{\beta} \prec Q$ holds. Thus, the subordination $q_{\beta} \prec Q$ holds if the inequalities

$$
Q(-1) \leq q_{\beta}(-1) \leq q_{\beta}(1) \leq Q(1)
$$

hold which in-turn yield a necessary condition for subordination $p \prec Q$. The inequalities $q_{\beta}(-1) \geq$ $Q(-1)$ and $q_{\beta}(1) \leq Q(1)$ yield $\beta \geq \beta_{1}=2(1-\log 2) / 1-e^{(1-e) / e}$ and $\beta \geq \beta_{2}=2(\sqrt{2}-1+$ $\log 2-\log (1+\sqrt{2})) /\left(e^{(1-e) / e}-1\right)$, respectively. Therefore, the subordination $q_{\beta} \prec Q$ holds if $\beta \geq$ $\max \left\{\beta_{1}, \beta_{2}\right\}=\beta_{1}$.

(c) The analytic function

$$
q_{\beta}(z)=\frac{2 \sqrt{\alpha} \beta+\log \frac{1+\sqrt{\alpha} z}{1-\sqrt{\alpha} z}}{2 \sqrt{\alpha} \beta}
$$

is a solution of the differential equation $\beta z q_{\beta}^{\prime}(z)=G_{\alpha}(z)-1$ in $\mathbb{D}$. Now computation shows that

$$
\mathcal{Q}(z)=z q_{\beta}^{\prime}(z) \psi\left(q_{\beta}(z)\right)=\frac{z}{1-\alpha z^{2}}
$$

is starlike in $\mathbb{D}$. Note that the function $h(z):=v(q(z))+\mathcal{Q}(z)=1+\mathcal{Q}(z)$ satisfies $\operatorname{Re}\left(z h^{\prime}(z) / \mathcal{Q}(z)\right)=$ $\operatorname{Re}\left(z \mathcal{Q}^{\prime}(z) / \mathcal{Q}(z)\right)>0$ in $\mathbb{D}$. Therefore, in view of the subordination relation 1 , the required subordination $p \prec Q$ holds if subordination $q_{\beta} \prec Q$. Similar to as in part (a), the desired subordination $p \prec Q$ holds if $\beta \geq \max \left\{\beta_{1}, \beta_{2}\right\}=\beta_{1}$, where $\beta_{1}=l(e) g(\alpha)$ and $\beta_{2}=-l(e) g(\alpha)$ such that

$$
g(\alpha)=\frac{1}{2 \sqrt{\alpha}} \log \frac{1+\sqrt{\alpha}}{1-\sqrt{\alpha}} .
$$


(d) Consider the analytic function

$$
q_{\beta}(z)=\frac{B \beta+(A-B) \log (1+B z)}{B \beta}
$$

which is a solution of differential equation

$$
\beta z q^{\prime}(z)=\frac{(A-B) z}{1+B z} .
$$

Since the function $(A-B) z /(1+B z)$ is starlike in $\mathbb{D}$, it follows that $\mathcal{Q}(z)=z q_{\beta}^{\prime}(z) \psi\left(q_{\beta}(z)\right)$ is starlike in $\mathbb{D}$. The function $h: \mathbb{D} \rightarrow \mathbb{C}$ defined by $h(z):=v\left(q_{\beta}(z)\right)+Q(z)=1+Q(z)$ satisfies $\operatorname{Re}\left(z h^{\prime}(z) / Q(z)\right)>0(z \in \mathbb{D})$. Thus, as in previous case, the subordination $p \prec \mathcal{Q}$ holds if $\beta \geq \max \left\{\beta_{1}, \beta_{2}\right\}=\beta_{1}$, where

$$
\beta_{1}=\frac{(A-B) \log (1-B)^{-1}}{B\left(1-e^{(1-e) / e}\right)} \quad \text { and } \quad \beta_{2}=\frac{(A-B) \log (1+B)}{B\left(e^{(1-e) / e}-1\right)} .
$$

(e) The differential equation

$$
\frac{d q}{d z}=\frac{\sin z}{\beta z}
$$

has an analytic solution

$$
q_{\beta}(z)=1+\frac{1}{\beta} \sum_{n=0}^{\infty} \frac{(-1)^{n} z^{2 n+1}}{(2 n+1) !(2 n+1)}
$$

in $\mathbb{D}$. Now the function $\mathcal{Q}(z)=z q_{\beta}^{\prime}(z) \psi\left(q_{\beta}(z)\right)=\sin z$ is starlike in $\mathbb{D}$ and the function $h(z):=$ $v(q(z))+\mathcal{Q}(z)=1+\mathcal{Q}(z)$, satisfies $\operatorname{Re}\left(z h^{\prime}(z) / \mathcal{Q}(z)\right)=\operatorname{Re}\left(z \mathcal{Q}^{\prime}(z) / \mathcal{Q}(z)\right)>0$ holds. As in part (a), the desired subordination $p(z) \prec Q(z)$ holds if $\beta \geq \max \left\{\beta_{1}, \beta_{2}\right\}=\beta_{1}$, where

$$
\beta_{1}=\frac{1}{\left(1-e^{(1-e) / e}\right)} \sum_{n=0}^{\infty} \frac{(-1)^{n}}{(2 n+1) !(2 n+1)} \approx 2.01905
$$

and

$$
\beta_{2}=\frac{1}{\left(e^{(e-1)}-1\right)} \sum_{n=0}^{\infty} \frac{(-1)^{n}}{(2 n+1) !(2 n+1)} \approx 0.206779 .
$$

(f) The differential equation

$$
\frac{d q}{d z}=\frac{z+\sqrt{1+z^{2}}-1}{\beta z}
$$

has an analytic solution

$$
q_{\beta}(z)=\frac{\beta+\left(z+\sqrt{1+z^{2}}-\log \left(1+\sqrt{1+z^{2}}\right)-1+\log 2\right)}{\beta} .
$$

Computation shows that the function

$$
\mathcal{Q}(z)=z q_{\beta}^{\prime}(z) \psi\left(q_{\beta}(z)\right)=z+\sqrt{1+z^{2}}-1
$$

is starlike in $\mathbb{D}$. As before, the function $h(z):=v(q(z))+\mathcal{Q}(z)$ satisfies $\operatorname{Re}\left(z h^{\prime}(z) / \mathcal{Q}(z)\right)>0, z \in \mathbb{D}$. Therefore, the desired subordination $p \prec Q$ holds if $\beta \geq \max \left\{\beta_{1}, \beta_{2}\right\}=\beta_{1}$, where

$$
\beta_{1}=\frac{2-\sqrt{2}-\log 2+\log (1+\sqrt{2})}{1-e^{(1-e) / e}} \approx 1.65198
$$


and

$$
\beta_{2}=\frac{\sqrt{2}+\log 2-\log (1+\sqrt{2})}{e^{(1-e) / e}-1} \approx 0.267979 .
$$

(g) The differential equation

$$
\frac{d q}{d z}=\frac{e^{z}-1}{\beta z}
$$

has an analytic solution

$$
q_{\beta}(z)=1+\frac{1}{\beta} \sum_{n=0}^{\infty} \frac{z^{n}}{n ! n} .
$$

Note that the function $\mathcal{Q}(z)=z q_{\beta}^{\prime}(z) \psi\left(q_{\beta}(z)\right)=e^{z}$ is starlike in the unit disk $\mathbb{D}$ and the function $h(z):=v(q(z))+\mathcal{Q}(z)=1+\mathcal{Q}(z)$ satisfies $\operatorname{Re}\left(z h^{\prime}(z) / \mathcal{Q}(z)\right)=\operatorname{Re}\left(z \mathcal{Q}^{\prime}(z) / \mathcal{Q}(z)\right)>0$. Now the subordination $p \prec Q$ holds if $\beta \geq \max \left\{\beta_{1}, \beta_{2}\right\}=\beta_{1}$, where

$$
\beta_{1}=\frac{1}{\left(1-e^{(1-e) / e}\right)} \sum_{n=0}^{\infty} \frac{(-1)^{n-1}}{n ! n} \approx 0.785166 \quad \text { and } \quad \beta_{2}=\frac{1}{\left(e^{(e-1)}-1\right)} \sum_{n=0}^{\infty} \frac{1}{n ! n} \approx 0.288069 .
$$

This ends the proof.

Theorem 1 also provides the following various sufficient conditions for the normalized analytic functions $f$ to be in the class $\mathcal{S}_{B}^{*}$.

Let function $f \in \mathcal{A}$ and set

$$
\mathrm{Y}_{\beta}\left(z, \frac{z f^{\prime}(z)}{f(z)}\right)=1+\beta \frac{z f^{\prime}(z)}{f(z)}\left(1-\frac{z f^{\prime}(z)}{f(z)}+\frac{z f^{\prime \prime}(z)}{f^{\prime}(z)}\right) .
$$

If either of the following subordination holds

(a) $\mathrm{Y}_{\beta}\left(z, \frac{z f^{\prime}(z)}{f(z)}\right) \prec \varphi_{0}(z)(\beta \geq 0.59533)$,

(b) $\mathrm{Y}_{\beta}\left(z, \frac{z f^{\prime}(z)}{f(z)}\right) \prec \sqrt{1+z}(\beta \geq 1.30984)$,

(c) $Y_{\beta}\left(z, \frac{f^{\prime}(z)}{f(z)}\right) \prec G_{\alpha}(z)\left(\beta \geq \frac{1}{\left(1-e^{(1-e) / e}\right)} \frac{1}{2 \sqrt{\alpha}} \log \frac{1+\sqrt{\alpha}}{1-\sqrt{\alpha}}\right)$,

(d) $\mathrm{Y}_{\beta}\left(z, \frac{f^{\prime}(z)}{f(z)}\right) \prec \frac{1+A z}{1+B z}\left(\beta \geq \frac{1}{\left(1-e^{(1-e) / e}\right)} \frac{A-B}{B} \log (1-B)^{-1}\right)$,

(e) $\mathrm{Y}_{\beta}\left(z, \frac{z f^{\prime}(z)}{f(z)}\right) \prec \varphi_{s}(z)(\beta \geq 2.01905)$,

(f) $\mathrm{Y}_{\beta}\left(z, \frac{z f^{\prime}(z)}{f(z)}\right) \prec \varphi_{q}(z)(\beta \geq 1.65198)$,

(g) $\mathrm{Y}_{\beta}\left(z, \frac{z f^{\prime}(z)}{f(z)}\right) \prec e^{z}(\beta \geq 0.785166)$,

then $f \in \mathcal{S}_{B}^{*}$.

The next result gives sharp lower bound on $\beta$ such that subordination $p \prec Q$ holds, whenever $1+\beta z p^{\prime}(z) / p(z) \prec \varphi_{0}(z)$ or $\varphi_{s}(z)$ or $\sqrt{1+z}$ or $G_{\alpha}(z)$ or $(1+A z) /(1+B z)$ or $\varphi_{s}(z)$ or $\varphi_{q}(z)$ or $e^{z}$.

Theorem 2. Let $0<\alpha<1,0<B<A<1$, and $p$ be an analytic function defined in $\mathbb{D}$ with $p(0)=1$. Set

$$
\Omega_{\beta}(z, p(z))=1+\beta \frac{z p^{\prime}(z)}{p(z)} .
$$

Then, the following conditions are sufficient for subordination $p \prec Q$.

(a) $\Omega_{\beta}(z, p(z)) \prec \varphi_{0}(z)$ for $\beta \geq \frac{e(2(1+\sqrt{2}) \log \sqrt{2}-1}{(e-1)(1+\sqrt{2})} \approx 0.441266$.

(b) $\Omega_{\beta}(z, p(z)) \prec \sqrt{1+z}$ for $\beta \geq \frac{2 e(1-\log 2)}{e-1} \approx 0.970868$.

(c) $\Omega_{\beta}(z, p(z)) \prec G_{\alpha}(z)$ for $\beta \geq \frac{e}{2(e-1) \sqrt{\alpha}} \log \frac{1+\sqrt{\alpha}}{1-\sqrt{\alpha}}$.

(d) $\Omega_{\beta}(z, p(z)) \prec \frac{1+A z}{1+B z}$ for $\beta \geq \frac{e}{B(e-1)}(A-B) \log (1-B)^{-1}$. 
(e) $\Omega_{\beta}(z, p(z)) \prec \varphi_{s}(z)$ for $\beta \geq \frac{e}{e-1} \sum_{n=0}^{\infty} \frac{(-1)^{n}}{(2 n+1) !(2 n+1)} \approx 1.49655$.

(f) $\Omega_{\beta}(z, p(z)) \prec \varphi_{q}(z)$ for $\beta \geq \frac{e}{e-1}(2-\sqrt{2}+\log (1+\sqrt{2})-\log 2) \approx 1.22447$.

(g) $\Omega_{\beta}(z, p(z)) \prec e^{z}$ for $\beta \geq \frac{1}{e-1} \sum_{n=0}^{\infty} \frac{1}{n ! n} \approx 0.766987$.

The lower bound on $\beta$ in each case is sharp.

Proof. Let us define $v(w)=1$ and $\psi(w)=\beta / w$ for all $w \in \mathbb{C}$.

(a) The function

$$
q_{\beta}(z)=\exp \left(-\frac{1}{\beta k}\left(z+2 k \log \left(1-\frac{z}{k}\right)\right)\right)
$$

satisfies the differential equation $\beta z q^{\prime}(z) / q(z)=\varphi_{0}(z)-1$. Clearly, the function $\mathcal{Q}: \overline{\mathbb{D}} \rightarrow$ defined by $\mathcal{Q}(z)=z q_{\beta}^{\prime}(z) \psi\left(q_{\beta}(z)\right)=\left(z-2 k^{2}+k\right) /(k-z)$ is starlike in $\mathbb{D}$. Further, the function $h(z):=$ $v\left(q_{\beta}(z)\right)+\mathcal{Q}(z)$ satisfies $\operatorname{Re}\left(z h^{\prime}(z) / \mathcal{Q}(z)\right)>0(z \in \mathbb{D})$. Thus, using Lemma 1, it follows that

$$
1+\beta \frac{z p^{\prime}(z)}{p(z)} \prec 1+\beta \frac{z q_{\beta}^{\prime}(z)}{q_{\beta}(z)} \text { implies } p \prec q_{\beta} \text {. }
$$

Now using Theorem 1 (a), the subordination $p \prec Q$ holds if $\beta \geq \max \left\{\beta_{1}, \beta_{2}\right\}=\beta_{1}$, where

$$
\beta_{1}=\frac{(-1+2(1+\sqrt{2}) \log \sqrt{2}) e}{(e-1)(1+\sqrt{2})}
$$

and

$$
\beta_{2}=-\frac{(1+2(1+\sqrt{2}) \log (2-\sqrt{2}))}{(e-1)(1+\sqrt{2})} .
$$

(b) The function

$$
q_{\beta}(z)=\exp \left(\frac{2}{\beta}(\sqrt{1+z}-\log (1+\sqrt{1+z})+\log 2-1)\right)
$$

is a solution of the differential equation

$$
\beta \frac{z q^{\prime}(z)}{q(z)}=\sqrt{1+z}-1
$$

Moreover, the function $\mathcal{Q}(z)=z q_{\beta}^{\prime}(z) \psi\left(q_{\beta}(z)\right)=\sqrt{1+z}-1$ is starlike in $\mathbb{D}$ and a computation shows that the function $h(z):=v(q(z))+\mathcal{Q}(z)$ satisfies $\operatorname{Re}\left(z h^{\prime}(z) / \mathcal{Q}(z)\right)>0(z \in \mathbb{D})$. Now the desired subordination $p \prec Q$ holds if $\beta \geq \max \left\{\beta_{1}, \beta_{2}\right\}=\beta_{1}$, where $\beta_{1}=2 e(1-\log 2) /(e-1)$ and $\beta_{2}=2(-1+\sqrt{2}+\log 2-\log (1+\sqrt{2})) /(e-1)$.

(c) Consider the function $q_{\beta}$ defined by

$$
q_{\beta}(z)=\exp \left(\frac{1}{2 \sqrt{\alpha} \beta} \log \frac{1+\sqrt{\alpha} z}{1-\sqrt{\alpha} z}\right) .
$$

It can be verified that the function $q_{\beta}$ is a solution of the differential equation

$$
\beta \frac{z q^{\prime}(z)}{q(z)}=\frac{1}{1-\alpha z^{2}}
$$

Now the function $\mathcal{Q}(z)=z q_{\beta}^{\prime}(z) \psi\left(q_{\beta}(z)\right)=1 /\left(1-\alpha z^{2}\right)$ is starlike in $\mathbb{D}$ and the function $h(z):=$ $v(q(z))+\mathcal{Q}(z)$ satisfies $\operatorname{Re}\left(z h^{\prime}(z) / \mathcal{Q}(z)\right)>0(z \in \mathbb{D})$. Now, as in previous cases, $p \prec Q$ holds only if $\beta \geq \max \left\{\beta_{1}, \beta_{2}\right\}=\beta_{1}$, where 


$$
\beta_{1}=\frac{e}{2(e-1) \sqrt{\alpha}} \log \frac{1+\sqrt{\alpha}}{1-\sqrt{\alpha}} \text { and } \beta_{2}=\frac{1}{2(e-1) \sqrt{\alpha}} \log \frac{1+\sqrt{\alpha}}{1-\sqrt{\alpha}} .
$$

(d) Let the function $q_{\beta}(z)=\exp ((A-B) \log (1+B z) / \beta B)$ be an analytic solution of the differential equation

$$
1+\beta \frac{z q^{\prime}(z)}{q(z)}=\frac{1+A z}{1+B z} .
$$

Now the desired subordination $p \prec Q$ holds if $\beta \geq \max \left\{\beta_{1}, \beta_{2}\right\}=\beta_{1}$, where $\beta_{1}=e(A-$ B) $\log (1-B)^{-1} / B(e-1)$ and $\beta_{2}=e(A-B) \log (1+B) / B(e-1)$.

(e) The differential equation $\beta z q^{\prime}(z) / q(z)=\sin z$ has an analytic solution given by

$$
q_{\beta}(z)=\exp \left(\frac{1}{\beta} \sum_{n=0}^{\infty} \frac{(-1)^{n} z^{2 n+1}}{(2 n+1) !(2 n+1)}\right) .
$$

As in part Theorem 2 (a), the subordination $p \prec Q$ holds if $\beta \geq \max \left\{\beta_{1}, \beta_{2}\right\}=\beta_{1}$ where

$$
\beta_{1}=\frac{e}{e-1} \sum_{n=0}^{\infty} \frac{(-1)^{n}}{(2 n+1) !(2 n+1)} \approx 1.49655
$$

and

$$
\beta_{2}=\frac{1}{e-1} \sum_{n=0}^{\infty} \frac{(-1)^{n}}{(2 n+1) !(2 n+1)} \approx 0.55055 .
$$

(f) The solution of the differential equation

$$
\frac{d q}{d z}=\frac{z+\sqrt{1+z^{2}}-1}{\beta z}
$$

is given by

$$
q_{\beta}(z)=\exp \left(\frac{z+\sqrt{1+z^{2}}-\log \left(1+\sqrt{1+z^{2}}\right)-1+\log 2}{\beta}\right) .
$$

As in proof of Theorem 2 (a), the desired result holds if $\beta \geq \max \left\{\beta_{1}, \beta_{2}\right\}=\beta_{1}$, where $\beta_{1}=$ $e(2-\sqrt{2}+\log (1+\sqrt{2})-\log 2) /(e-1)$ and $\beta_{2}=(\sqrt{2}-\log (1+\sqrt{2})+\log 2) /(e-1)$.

(g) The differential equation $\beta z q^{\prime}(z) / q(z)=e^{z}-1$ has a solution

$$
q_{\beta}(z)=\exp \left(\frac{1}{\beta} \sum_{n=1}^{\infty} \frac{z^{n}}{n ! n}\right)
$$

analytic in $\mathbb{D}$. Thus, as previous, the subordination $p \prec Q$ holds if $\beta \geq \max \left\{\beta_{1}, \beta_{2}\right\}=\beta_{2}$, where

$$
\beta_{1}=\frac{e}{e-1} \sum_{n=0}^{\infty} \frac{(-1)^{n-1}}{n ! n} \approx 0.581976 \quad \text { and } \quad \beta_{2}=\frac{1}{e-1} \sum_{n=0}^{\infty} \frac{1}{n ! n} \approx 0.766987 .
$$

This ends the proof.

Next, Theorem 2 also provides the following various sufficient conditions for the normalized analytic functions $f$ to be in the class $\mathcal{S}_{B}^{*}$. Let the function $f \in \mathcal{A}$ and set

$$
\Omega_{\beta}\left(z, \frac{z f^{\prime}(z)}{f(z)}\right)=1+\beta\left(1-\frac{z f^{\prime}(z)}{f(z)}+\frac{z f^{\prime \prime}(z)}{f^{\prime}(z)}\right) .
$$

If either of the following subordination conditions are fulfilled: 
(a) $\Omega_{\beta}\left(z, \frac{z f^{\prime}(z)}{f(z)}\right) \prec \varphi_{0}(z)(\beta \geq 0.441266)$,

(b) $\Omega_{\beta}\left(z, \frac{z f^{\prime}(z)}{f(z)}\right) \prec \sqrt{1+z}(\beta \geq 0.970868)$,

(c) $\Omega_{\beta}\left(z, \frac{z f^{\prime}(z)}{f(z)}\right) \prec G_{\alpha}(z)\left(\beta \geq \frac{e}{2(e-1) \sqrt{\alpha}} \log \frac{1+\sqrt{\alpha}}{1-\sqrt{\alpha}}\right)$,

(d) $\Omega_{\beta}\left(z, \frac{z f^{\prime}(z)}{f(z)}\right) \prec \frac{1+A z}{1+B z}\left(\beta \geq \frac{e}{B(e-1)}(A-B) \log (1-B)^{-1}\right)$,

(e) $\Omega_{\beta}\left(z, \frac{z f^{\prime}(z)}{f(z)}\right) \prec \varphi_{s}(z)(\beta \geq 1.49655)$,

(f) $\Omega_{\beta}\left(z, \frac{z f^{\prime}(z)}{f(z)}\right) \prec \varphi_{q}(z)(\beta \geq 1.22447)$,

(g) $\Omega_{\beta}\left(z, \frac{z f^{\prime}(z)}{f(z)}\right) \prec e^{z}(\beta \geq 0.766987)$,

then $f \in \mathcal{S}_{B}^{*}$.

In the following theorem, the sharp lower bound on $\beta$ is obtained so that the subordination $p \prec Q$ holds, whenever $1+\beta z p^{\prime}(z) / p^{2}(z) \prec \varphi_{0}(z)$ or $\varphi_{s}(z)$ or $\sqrt{1+z}$ or $G_{\alpha}(z)$ or $(1+A z) /(1+B z)$ or $\varphi_{s}(z)$ or $\varphi_{q}(z)$ or $e^{z}$. These results can be proved by defining the functions $v, \psi: \mathbb{D} \rightarrow$ defined by $v(w)=1$ and $\psi(w)=\beta / w^{2}$ and proceeding in a similar fashion as in the proofs of Theorems 1 and 2.

Theorem 3. Let $0<\alpha<1,0<B<A<1$, and $p$ be an analytic function defined in $\mathbb{D}$ with $p(0)=1$.

Set

$$
\Xi_{\beta}(z, p(z))=1+\beta \frac{z p^{\prime}(z)}{p^{2}(z)} .
$$

Then, the following conditions are sufficient for $p \prec Q$.

(a) $\Xi_{\beta}(z, p(z)) \prec \varphi_{0}(z)$ for $\beta \geq \frac{1+2(\sqrt{2}+1) \log (2-\sqrt{2})}{(1+\sqrt{2})\left(e^{(1-e)}-1\right)} \approx 0.798642$.

(b) $\Xi_{\beta}(z, p(z)) \prec \sqrt{1+z}$ for $\beta \geq \frac{2(-1+\sqrt{2}+\log 2-\log (1+\sqrt{2}))}{1-e^{1-e}} \approx 0.550768$.

(c) $\Xi_{\beta}(z, p(z)) \prec G_{\alpha}(z)$ for $\beta \geq \frac{e^{e-1}}{e^{e-1}-1} \frac{1}{2 \sqrt{\alpha}} \log \frac{1+\sqrt{\alpha}}{1-\sqrt{\alpha}}$.

(d) $\Xi_{\beta}(z, p(z)) \prec \frac{1+A z}{1+B z}$ for $\beta \geq \frac{e^{(1-e) / e}}{1-e^{(1-e) / e}} \frac{(A-B) \log (1-B)^{-1}}{B}$.

(e) $\Xi_{\beta}(z, p(z)) \prec \varphi_{s}(z)$ for $\beta \geq \frac{e^{e-1}}{e^{e-1}-1} \sum_{n=0}^{\infty} \frac{(-1)^{n}}{(2 n+1) !(2 n+1)} \approx 1.15278$.

(f) $\Xi_{\beta}(z, p(z)) \prec \varphi_{q}(z)$ for $\beta \geq \frac{e^{e-1}}{e^{e-1}-1}(\sqrt{2}-\log (1+\sqrt{2})+\log 2) \approx 1.49397$.

(g) $\Xi_{\beta}(z, p(z)) \prec e^{z}$ for $\beta \geq \frac{e^{e-1}}{e^{e-1}-1} \sum_{n=0}^{\infty} \frac{1}{n ! n} \approx 1.60597$.

The lower bound on $\beta$ in each case is sharp.

Let $f \in \mathcal{A}$ and set

$$
\Xi_{\beta}\left(z, \frac{z f^{\prime}(z)}{f(z)}\right)=1+\beta\left(\frac{z f^{\prime}(z)}{f(z)}\right)^{-1}\left(1-\frac{z f^{\prime}(z)}{f(z)}+\frac{z f^{\prime \prime}(z)}{f^{\prime}(z)}\right) .
$$

If either of the following subordination holds

(a) $\Xi_{\beta}\left(z, \frac{z f^{\prime}(z)}{f(z)}\right) \prec \varphi_{0}(z)(\beta \geq 0.798642)$,

(b) $\Xi_{\beta}\left(z, \frac{z f^{\prime}(z)}{f(z)}\right) \prec \sqrt{1+z}(\beta \geq 0.550768)$,

(c) $\Xi_{\beta}\left(z, \frac{z f^{\prime}(z)}{f(z)}\right) \prec G_{\alpha}(z)\left(\beta \geq \frac{e^{e-1}}{e^{e-1}-1} \frac{1}{2 \sqrt{\alpha}} \log \frac{1+\sqrt{\alpha}}{1-\sqrt{\alpha}}\right)$,

(d) $\Xi_{\beta}\left(z, \frac{z f^{\prime}(z)}{f(z)}\right) \prec \frac{1+A z}{1+B z}\left(\beta \geq \frac{e^{(1-e) / e}}{1-e^{(1-e) / e}} \frac{(A-B) \log (1-B)^{-1}}{B}\right)$,

(e) $\Xi_{\beta}\left(z, \frac{z f^{\prime}(z)}{f(z)}\right) \prec \varphi_{s}(z)(\beta \geq 1.15278)$,

(f) $\Xi_{\beta}\left(z, \frac{z f^{\prime}(z)}{f(z)}\right) \prec \varphi_{q}(z)(\beta \geq 1.49397)$,

(g) $\Xi_{\beta}\left(z, \frac{z f^{\prime}(z)}{f(z)}\right) \prec e^{z}(\beta \geq 1.60597)$,

then $f \in \mathcal{S}_{B}^{*}$. 


\section{Radius Estimates}

Let $\theta_{1}$ and $\theta_{2}$ be two sub-families of $\mathcal{A}$. The $\theta_{1}$ radius of $\theta_{2}$ is the largest number $\rho \in(0,1)$ such that $r^{-1} f(r z) \in \theta_{1}, 0<r \leq \rho$ for all $f \in \theta_{2}$. Grunsky [34] obtained the radius of starlikeness for functions in the class $\mathcal{S}$. Sokół [35] computed the radius of $\alpha$-convexity and $\alpha$-starlikeness for a class $\mathcal{S}_{L}^{*}$. In 2016, authors [7] determined the $\mathcal{S}_{R}^{*}$-radius for various subclasses of starlike functions. For more results on radius problems, see [36-41].

The main technique involved in tackling the $\mathcal{S}_{B}^{*}$-radius estimates for classes of functions $f$ is the determination of the disk that contains the values of $z f^{\prime}(z) / f(z)$. The associated technical lemma is achieved as:

Lemma 2. Let $Q(z):=e^{e^{z}-1}, z \in \mathbb{D}$. Define the function $r:\left[e^{1 / e-1}, e^{e-1}\right] \rightarrow \mathbb{R}^{+}$by

$$
r(a):= \begin{cases}\frac{e a-e^{1 / e}}{e}, & e^{\frac{1}{e}-1} \leq a \leq \frac{e^{1 / e}+e^{e}}{2 e} \\ \frac{e^{e}-e a}{e}, & \frac{e^{1 / e}+e^{e}}{2 e} \leq a \leq e^{e-1}\end{cases}
$$

Then, the following holds:

$$
\{w \in \mathbb{C}:|w-a|<r(a)\} \subset \Omega_{B} \subset\left\{w \in \mathbb{C}:|w-1|<\frac{e^{e}-e}{e}\right\} .
$$

Proof. To prove the assertion, we let $z=e^{i t}, t \in(-\pi, \pi]$. Therefore,

$$
Q\left(e^{i t}\right)=e^{e^{i t}-1}=u(t)+i v(t)
$$

with

$$
u(t):=\cos \left(\sin (\sin t) e^{\cos t}\right) \exp \left(e^{\cos t} \cos (\sin t)-1\right)
$$

and

$$
v(t):=\sin \left(\sin (\sin t) e^{\cos t}\right) \exp \left(e^{\cos (t)} \cos (\sin t)-1\right)
$$

Now, consider the square of the distance of an arbitrary point $(u(t), v(t))$ on the boundary of $\partial Q(\mathbb{D})$ from $(a, 0)$ and is given by

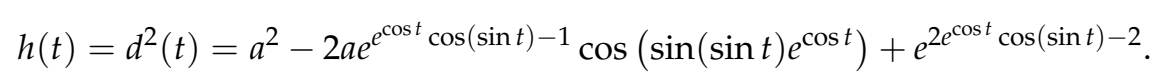

Now we need to prove $|w-a|<r(a)$ is the largest disk contained in $Q(\mathbb{D})$. For this, we need to show that $\min _{-\pi \leq t \leq \pi} d(t)=r(a)$. Since $h$ is an even function, i.e., $h(t)=h(-t)$, we need to only consider the case when $t \in[0, \pi]$. Now $h^{\prime}(t)=0$ has three roots viz. $0, \pi$ and $t_{0}(a) \in(0, \pi)$. Among these roots, the root $t_{0}(a)$ depends on $a$ and graphics reveals that $h$ is increasing in the interval $\left[0, t_{0}(a)\right]$ and decreasing in $\left[t_{0}(a), \pi\right]$, and therefore, $h$ attains its minimum either at 0 or $\pi$. Further computations give $h(\pi)=\left(e a-e^{1 / e}\right)^{2} / e^{2}$ and $h(0)=\left(e^{e}-e a\right)^{2} / e^{2}$. Hence, we have

$$
\min _{-\pi \leq t \leq \pi} h(t)=\min \{h(0), h(\pi)\}= \begin{cases}h(\pi), & e^{\frac{1}{e}-1} \leq a \leq \frac{e^{1 / e}+e^{e}}{2 e} \\ h(0), & \frac{e^{1 / e}+e^{e}}{2 e} \leq a \leq e^{e-1}\end{cases}
$$

Therefore, we can write

$$
\min _{-\pi \leq t \leq \pi} d(t)= \begin{cases}\frac{e a-e^{1 / e}}{e}, & e^{\frac{1}{e}-1} \leq a \leq \frac{e^{1 / e}+e^{e}}{2 e} \\ \frac{e^{e}-e a}{e}, & \frac{e^{1 / e}+e^{e}}{2 e} \leq a \leq e^{e-1}\end{cases}
$$


To find the circle of minimum radius with center at $(1,0)$ containing the domain $Q(\mathbb{D})$, we need to find the maximum distance from $(1,0)$ to an arbitrary point on the boundary of the domain $Q(\mathbb{D})$. The square of this distance function is given by

$$
\phi(t)=-2 e^{e^{\cos t} \cos (\sin t)-1} \cos \left(\sin (\sin t) e^{\cos t}\right)+e^{2 e^{\cos t} \cos (\sin t)-2}+1 .
$$

The equation $\phi^{\prime}(t)=0$ has two roots in $[0, \pi]$, namely 0 and $\pi$. It is easy to see that $\phi(0)=\left(e-e^{e}\right)^{2} / e^{2}$ and $\phi(\pi / 2)=\left(e-e^{1 / e}\right)^{2} / e^{2}$. Therefore,

$$
\max \{\phi(0), \phi(\pi)\}=\phi(0)=\frac{\left(e-e^{e}\right)^{2}}{e^{2}} .
$$

Hence, the radius of the smallest disk containing $Q(\mathbb{D})$ is $\left(e-e^{e}\right) / e$. This ends the proof.

We now recall some classes and results related to them which are to be used for further development of this section. For $-1 \leq B<A \leq 1$, let

$$
\mathcal{P}_{n}[A, B]:=\left\{p(z)=1+\sum_{k=n}^{\infty} c_{n} z^{n}: p(z) \prec \frac{1+A z}{1+B z}\right\} .
$$

Let us denote $\mathcal{P}_{n}(\alpha):=\mathcal{P}_{n}[1-2 \alpha,-1]$ and $\mathcal{P}_{1}(0)=: \mathcal{P}$. For $f \in \mathcal{A}$, if we set $p(z)=z f^{\prime}(z) / f(z)$ and $p(z)=1+z f^{\prime \prime}(z) / f^{\prime}(z)$, then the class $\mathcal{P}[A, B]$ is denoted by $\mathcal{S}^{*}[A, B]$ and $\mathcal{K}[A, B]$, respectively. These classes were introduced and studied by [2]. Further, let $\mathcal{S}^{*}(\alpha):=\mathcal{S}^{*}[1-2 \alpha,-1]$.

The following results will be needed:

Lemma 3. [42] If $p \in \mathcal{P}_{n}[A, B]$, then, for $|z|=r$,

$$
\left|p(z)-\frac{1-A B r^{2 n}}{1-B^{2} r^{2 n}}\right| \leq \frac{(A-B) r^{n}}{1-B^{2} r^{2 n}}
$$

In particular, if $p \in \mathcal{P}_{n}(\alpha)$, then, for $|z|=r$,

$$
\left|p(z)-\frac{(1+(1-2 \alpha)) r^{2 n}}{1-r^{2 n}}\right| \leq \frac{2(1-\alpha) r^{n}}{1-r^{2 n}} .
$$

Lemma 4. [43] If $p \in \mathcal{P}_{n}(\alpha)$, then, for $|z|=r$,

$$
\left|\frac{z p^{\prime}(z)}{p(z)}\right| \leq \frac{2(1-\alpha) n r^{n}}{\left(1-r^{n}\right)\left(1+(1-2 \alpha) r^{n}\right)} .
$$

The main objective of this section is to determine the $\mathcal{S}_{B}^{*}$-radii constants for functions belonging to certain well-known subclasses of $\mathcal{A}$. Let $\mathcal{G}$ denote the class of functions $f \in \mathcal{S}$ for which $f(z) / z \in \mathcal{P}$. The following theorem gives the sharp $\mathcal{S}_{B}^{*}$-radius for the class $\mathcal{G}$.

Theorem 4. Let $f \in \mathcal{G}$. Then, the sharp $\mathcal{S}_{B}^{*}$-radius is

$$
R_{\mathcal{S}_{B}^{*}}(\mathcal{G}):=\frac{e-e^{1 / e}}{\sqrt{2 e^{2}-2 e^{1+\frac{1}{e}}+e^{2 / e}}+e} \approx 0.222654 .
$$

Proof. Since $f \in \mathcal{G}$, therefore, $f(z) / z \in \mathcal{P}$. Then, from Lemma 2, we must have

$$
\left|\frac{z f^{\prime}(z)}{f(z)}-1\right| \leq \frac{2 r}{1-r^{2}}
$$


Therefore, $f \in \mathcal{S}_{B}^{*}$ if $2 r /\left(1-r^{2}\right) \leq\left(e-e^{1 / e}\right) / e$, or equivalently if

$$
\left(e-e^{1 / e}\right) r^{2}+2 e r+e^{1 / e}-e \leq 0
$$

which holds for all

$$
r \leq \frac{e-e^{1 / e}}{\sqrt{2 e^{2}-2 e^{1+\frac{1}{e}}+e^{2 / e}}+e}=: R_{\mathcal{S}_{B}^{*}}(\mathcal{G}) \approx 0.222654 .
$$

For verification of sharpness, consider the function $f(z)=z(1+z) /(1-z)$. Then, $f(z) / z \in \mathcal{P}$ and at $z=R_{\mathcal{S}_{B}^{*}}(\mathcal{G})$, we have

$$
\frac{R_{\mathcal{S}_{B}^{*}}(\mathcal{G}) f^{\prime}\left(R_{\mathcal{S}_{B}^{*}}(\mathcal{G})\right)}{f\left(R_{\mathcal{S}_{B}^{*}}(\mathcal{G})\right)}-1=\frac{R_{\mathcal{S}_{B}^{*}}(\mathcal{G})}{1-R_{\mathcal{S}_{B}^{*}}(\mathcal{G})}=1-e^{\frac{1}{e}-1} .
$$

Hence the result is sharp.

In the following theorem, we shall investigate sharp $\mathcal{S}_{B}^{*}$-radius for the class $\mathcal{S}^{*}[A, B]$.

Theorem 5. Let $f \in \mathcal{S}^{*}[A, B]$. Then,

1. for $0 \leq B<A \leq 1$, the sharp $\mathcal{S}_{B}^{*}$-radius for the class $\mathcal{S}^{*}[A, B]$ is

$$
R_{\mathcal{S}_{B}^{*}}\left(\mathcal{S}^{*}[A, B]\right)=\min \left\{1 ; \frac{\sqrt{e-e^{1 / e}}}{\sqrt{e A B-e^{1 / e} B^{2}}} ; \frac{e^{1 / e}-e}{e^{1 / e} B-e A}\right\} .
$$

2. for $-1 \leq B<0 \leq A \leq 1$, the sharp $\mathcal{S}_{B}^{*}$-radius for the class $\mathcal{S}^{*}[A, B]$ is

$$
R_{\mathcal{S}_{B}^{*}}\left(\mathcal{S}^{*}[A, B]\right)=\min \left\{1 ; \sqrt{\frac{-2 e+e^{1 / e}+e^{e}}{-2 e A B+e^{1 / e} B^{2}+e^{e} B^{2}}} ; \frac{e^{1 / e}-e}{e^{1 / e} B-e A}\right\} .
$$

Proof. Let $f \in \mathcal{S}^{*}[A, B]$. Then using Lemma 4 , we see that $f$ maps the disk $|z| \leq r$ onto the disk

$$
\left|\frac{z f^{\prime}(z)}{f(z)}-\frac{1-A B r^{2}}{1-B^{2} r^{2}}\right| \leq \frac{(A-B) r}{1-B^{2} r^{2}}
$$

The center of the above disk is at $(c, 0)$ and the radius is $R$, where

$$
c:=\frac{1-A B r^{2}}{1-B^{2} r^{2}} \text { and } R:=\frac{(A-B) r}{1-B^{2} r^{2}} .
$$

(1) We see that $c \leq\left(e^{1 / e}+e^{e}\right) /(2 e)$ holds for all $0 \leq B<A \leq 1$ and $0<r<1$. Further, the condition $1-e^{1 / e} \leq c$ is equivalent to

$$
-e A B r^{2}+e^{1 / e} B^{2} r^{2}-e^{1 / e}+e \geq 0
$$

which holds for all

$$
r \leq \sqrt{\frac{e-e^{1 / e}}{e A B-e^{1 / e} B^{2}}}=: r_{1} .
$$

Further computation shows that the condition $R \leq\left(e^{e} a-e^{1 / e}\right) / e$ is equivalent to $e A r-e^{1 / e} B r+$ $e^{1 / e}-e \leq 0$ which holds for all

$$
r \leq \frac{e^{1 / e}-e}{e^{1 / e} B-e A}=: r_{2}
$$

Now from Lemma $2, f \in \mathcal{S}_{B}^{*}$ for all $|z| \leq R_{\mathcal{S}_{B}^{*}}\left(\mathcal{S}^{*}[A, B]\right)=\min \left\{1 ; r_{1} ; r_{2}\right\}$. 
(2) Let $-1 \leq B<0 \leq A \leq 1$. Then we see that $e^{1 / e-1} \leq c$ holds for all $0<r<1$. Further, $c \leq\left(e^{e}+e^{1 / e}\right) / 2 e$ is equivalent to

$$
-2 e A B r^{2}+e^{1 / e} B^{2} r^{2}+e^{e} B^{2} r^{2}-e^{1 / e}-e^{e}+2 e \leq 0
$$

which holds for

$$
r \leq \sqrt{\frac{-2 e+e^{1 / e}+e^{e}}{-2 e A B+e^{1 / e} B^{2}+e^{e} B^{2}}}=: r_{3} .
$$

Now, as in the previous case $R<\left(e c-e^{1 / e}\right) / e$ holds if $r \leq r_{2}$. Therefore, $\mathcal{S}_{B}^{*}$-radius for the class $\mathcal{S}^{*}[A, B]$ is $R_{\mathcal{S}_{B}^{*}}\left(\mathcal{S}^{*}[A, B]\right)=\min \left\{1 ; r_{2} ; r_{3}\right\}$.

The equality holds in case of the function $f_{0}$ defined by

$$
f_{0}(z)= \begin{cases}z(1+B z)^{\frac{A}{B}-1}, & B \neq 0 \\ z e^{A z}, & B=0\end{cases}
$$

This ends the proof.

Remark 1. Let $f \in \mathcal{S}^{*}$. Then, since $\mathcal{S}^{*}=\mathcal{S}^{*}[0,-1]$, it follows from the above theorem, that the $\mathcal{S}_{B}^{*}$-radius for starlike functions is $r_{4}:=\left(e-e^{1 / e}\right) /\left(e+e^{1 / e}\right) \approx 0.30594$. To see the sharpness, consider the Koebe function $k(z)=z /(1-z)^{2}$. Then, at $z=r_{4}$, we have

$$
\frac{r_{4} f^{\prime}\left(r_{4}\right)}{f\left(r_{4}\right)}=\frac{1+r_{4}}{1-r_{4}}=e^{1-\frac{1}{e}} .
$$

Because the function $k$ is univalent too, it follows that the $\mathcal{S}_{B}^{*}$-radius for the class $\mathcal{S}$ and $\mathcal{S}^{*}$ is $r_{4}$. Therefore, the radius $r_{4}$ can not be increased. Thus, we have the following:

Corollary 1. The sharp $\mathcal{S}_{B}^{*}$-radius for the classes $\mathcal{S}$ and $\mathcal{S}^{*}$ is $\left(e-e^{1 / e}\right) /\left(e+e^{1 / e}\right) \approx 0.30594$.

Let the class $\mathcal{F}_{1}$ be defined by

$$
\mathcal{F}_{1}:=\left\{f \in \mathcal{A}: \operatorname{Re} \frac{f(z)}{g(z)}>0 \text { and } \operatorname{Re} \frac{g(z)}{z}>0, g \in \mathcal{A}\right\} .
$$

The following theorem gives the sharp $\mathcal{S}_{B}^{*}$-radius for the class $\mathcal{F}_{1}$.

Theorem 6. Let $f \in \mathcal{F}_{1}$. Then, the sharp $\mathcal{S}_{B}^{*}$-radius is

$$
R_{\mathcal{S}_{B}^{*}}\left(\mathcal{F}_{1}\right)=\frac{e-e^{1 / e}}{\sqrt{5 e^{2}-2 e^{1+\frac{1}{e}}+e^{2 / e}}+2 e} \approx 0.11557
$$

Proof. Since $f \in \mathcal{F}_{1}$, there is $g \in \mathcal{A}$ such that $\operatorname{Re}(g(z) / z)>0$. Define the functions $p, h: \mathbb{D} \rightarrow \mathbb{C}$ by

$$
p(z)=\frac{g(z)}{z} \text { and } h(z)=\frac{f(z)}{g(z)}
$$

Then, through some assumptions, we have $p, h \in \mathcal{P}$. Now using Lemma 4 , we get

$$
\begin{aligned}
\left|\frac{z f^{\prime}(z)}{f(z)}-1\right| & \leq\left|\frac{z h^{\prime}(z)}{h(z)}\right|+\left|\frac{z p^{\prime}(z)}{p(z)}\right| \\
& \leq \frac{4 r}{1-r^{2}} \leq \frac{e-e^{1 / e}}{e}
\end{aligned}
$$


this holds if and only if $\left(e-e^{1 / e}\right) r^{2}+4 e r+e^{1 / e}-e \leq 0$, that is if

$$
r \leq \frac{e-e^{1 / e}}{\sqrt{5 e^{2}-2 e^{1+\frac{1}{e}}+e^{2 / e}}+2 e}=: R_{\mathcal{S}_{B}^{*}}\left(\mathcal{F}_{1}\right) \approx 0.11557 .
$$

Consider the functions $f_{2}$ and $g_{2}$ defined by

$$
f_{2}(z)=z\left(\frac{1+z}{1-z}\right)^{2} \text { and } g_{2}(z)=z\left(\frac{1+z}{1-z}\right) .
$$

Further, we have $\operatorname{Re}\left(f_{2}(z) / g_{2}(z)\right)>0$ and $\operatorname{Re}\left(g_{2}(z) / z\right)>0$, and therefore $f \in \mathcal{F}_{1}$. Now a computation shows that, for $z=R_{\mathcal{S}_{B}^{*}}\left(\mathcal{F}_{1}\right)$,

$$
\frac{R_{\mathcal{S}_{B}^{*}}\left(\mathcal{F}_{1}\right) f_{2}^{\prime}\left(R_{\mathcal{S}_{B}^{*}}\left(\mathcal{F}_{1}\right)\right)}{f_{2}\left(R_{\mathcal{S}_{B}^{*}}\left(\mathcal{F}_{1}\right)\right)}-1=\frac{4 R_{\mathcal{S}_{B}^{*}}\left(\mathcal{F}_{1}\right)}{1-R_{\mathcal{S}_{B}^{*}}\left(\mathcal{F}_{1}\right)^{2}}=1-e^{\frac{1}{e}-1} .
$$

Hence the result is sharp.

Let us define the class $\mathcal{F}_{2}$ by

$$
\mathcal{F}_{2}:=\left\{f \in \mathcal{A}: \operatorname{Re} \frac{f(z)}{g(z)}>0 \text { and } \operatorname{Re} \frac{g(z)}{z}>1 / 2, g \in \mathcal{A}\right\} .
$$

The following theorem gives the sharp $\mathcal{S}_{B}^{*}$-radius for the class $\mathcal{F}_{2}$.

Theorem 7. Let $f \in \mathcal{F}_{2}$. Then, the sharp $\mathcal{S}_{B}^{*}$-radius is

$$
\mathcal{S}_{B}^{*}\left(\mathcal{F}_{2}\right)=\frac{2\left(e-e^{1 / e}\right)}{\sqrt{17 e^{2}-12 e^{1+\frac{1}{e}}+4 e^{2 / e}}+3 e} \approx 0.145776
$$

Proof. Since $f \in \mathcal{F}_{2}$ and $g \in \mathcal{A}$ satisfies $\operatorname{Re}(g(z) / z)>1 / 2$. Now define the functions $p, h: \mathbb{D} \rightarrow \mathbb{C}$ by $p(z)=g(z) / z$ and $h(z)=f(z) / g(z)$. Then, it is clear that $p \in \mathcal{P}(1 / 2)$ and $h \in \mathcal{P}$. Further, since $f(z)=z p(z) h(z)$, it follows from Lemma 4 , get

$$
\left|\frac{z f^{\prime}(z)}{f(z)}-1\right| \leq \frac{3 r+r^{2}}{1-r^{2}} \leq \frac{e-e^{1 / e}}{e}
$$

provided $-e^{1 / e} r^{2}+2 e r^{2}+3 e r+e^{1 / e}-e \leq 0$. This holds for

$$
r \leq \frac{2\left(e-e^{1 / e}\right)}{\sqrt{17 e^{2}-12 e^{1+\frac{1}{e}}+4 e^{2 / e}}+3 e}=: \mathcal{S}_{B}^{*}\left(\mathcal{F}_{2}\right) \approx 0.145776
$$

Thus, $f \in \mathcal{S}_{B}^{*}$ for $r \leq \mathcal{S}_{B}^{*}\left(\mathcal{F}_{2}\right)$.

For the sharpness of the result, consider the functions

$$
f_{3}(z)=\frac{z(1+z)}{(1-z)^{2}} \text { and } g_{3}(z)=\frac{z}{1-z}
$$

Then, we see that $\operatorname{Re}\left(f_{3}(z) / g_{3}(z)\right)>0$ and $\operatorname{Re}\left(g_{3}(z) / z\right)>1 / 2$, and therefore, $f \in \mathcal{F}_{2}$. Now from the definition of $f_{0}$, we see that at $z=\mathcal{S}_{B}^{*}\left(\mathcal{F}_{2}\right)$, 


$$
\frac{\mathcal{S}_{B}^{*}\left(\mathcal{F}_{2}\right) f_{3}^{\prime}\left(\mathcal{S}_{B}^{*}\left(\mathcal{F}_{2}\right)\right)}{f_{3}\left(\mathcal{S}_{B}^{*}\left(\mathcal{F}_{2}\right)\right)}-1=\frac{3 \mathcal{S}_{B}^{*}\left(\mathcal{F}_{2}\right)+\mathcal{S}_{B}^{*}\left(\mathcal{F}_{2}\right)^{2}}{1-\mathcal{S}_{B}^{*}\left(\mathcal{F}_{2}\right)^{2}}=1-e^{\frac{1}{e}-1}
$$

This confirms the sharpness of the result.

Define the class $\mathcal{F}_{3}$ by

$$
\mathcal{F}_{3}:=\left\{f \in \mathcal{A}:\left|\frac{f(z)}{g(z)}-1\right|<1 \text { and } \operatorname{Re} \frac{g(z)}{z}>0, g \in \mathcal{A}\right\} .
$$

The next result gives the sharp $\mathcal{S}_{B}^{*}$-radius for the class $\mathcal{F}_{3}$.

Theorem 8. Let $f \in \mathcal{F}_{3}$. Then, the sharp $\mathcal{S}_{B}^{*}$-radius is

$$
\mathcal{S}_{B}^{*}\left(\mathcal{F}_{3}\right)=\frac{2\left(e-e^{1 / e}\right)}{\sqrt{17 e^{2}-12 e^{1+\frac{1}{e}}+4 e^{2 / e}}+3 e} \approx 0.145776
$$

Proof. Since $f \in \mathcal{F}_{3}$, it follows that $p \in \mathcal{P}$ and $h \in \mathcal{P}(1 / 2)$, where the functions $p, h: \mathbb{D} \rightarrow \mathbb{C}$ are defined by $p(z)=g(z) / z$ and $h(z)=g(z) / f(z)$. Now since $f(z)=z p(z) / h(z)$ from Lemma 4 , we have

$$
\left|\frac{z f^{\prime}(z)}{f(z)}-1\right| \leq \frac{3 r+r^{2}}{1-r^{2}} \leq \frac{e-e^{1 / e}}{e}
$$

which holds for all $r \leq \mathcal{S}_{B}^{*}\left(\mathcal{F}_{3}\right)$.

Consider the functions $f_{4}$ and $g_{4}$ defined by

$$
f_{4}(z)=\frac{z(1+z)^{2}}{(1-z)} \text { and } g_{4}(z)=\frac{z(1+z)}{1-z} .
$$

The results are sharp, since at $z=\mathcal{S}_{B}^{*}\left(\mathcal{F}_{3}\right)$, we have

$$
\frac{\mathcal{S}_{B}^{*}\left(\mathcal{F}_{3}\right) f_{4}^{\prime}\left(\mathcal{S}_{B}^{*}\left(\mathcal{F}_{3}\right)\right)}{f_{4}\left(\mathcal{S}_{B}^{*}\left(\mathcal{F}_{3}\right)\right)}=2-e^{\frac{1}{e}-1}
$$

This completes the proof.

Author Contributions: All authors contributed equally.

Funding: This research was funded by the Basic Science Research Program through the National Research Foundation of Korea (NRF) funded by the Ministry of Education, Science and Technology (No. 2016R1D1A1A09916450).

Conflicts of Interest: The authors declare no conflict of interest

\section{References}

1. Ma, W.C.; Minda, D. A unified treatment of some special classes of univalent functions. In Proceedings of the Conference on Complex Analysis, Tianjin, China, 19-23 June 1992.

2. Janowski, W. Extremal problems for a family of functions with positive real part and for some related families. Ann. Polon. Math. 1970, 23, 159-177. [CrossRef]

3. Uralegaddi, B.A.; Ganigi, M.D.; Sarangi, S.M. Univalent functions with positive coefficients. Tamkang J. Math. 1994, 25, 225-230.

4. Sokól, J.; Stankiewicz, J. Radius of convexity of some subclasses of strongly starlike functions. Zeszyty Nauk. Politech. Rzeszowskiej Mat. 1996, 19, 101-105.

5. Raina, R.K.; Sokół, J. Some properties related to a certain class of starlike functions. C. R. Math. Acad. Sci. 2015, 353, 973-978. [CrossRef] 
6. Mendiratta, R.; Nagpal, S.; Ravichandran, V. On a subclass of strongly starlike functions associated with exponential function. Bull. Malays. Math. Sci. Soc. 2015, 38, 365-386. [CrossRef]

7. Kumar, S.; Ravichandran, V. A subclass of starlike functions associated with a rational function. Southeast Asian Bull. Math. 2016, 40, 199-212.

8. Cho, N.E.; Kumar, V.; Kumar, S.S.; Ravichandran, V. Radius problems for starlike functions associated with the Sine function. Bull. Iran. Math. Soc. 2018. [CrossRef]

9. Kargar, R.; Ebadian, A.; Sokół, J. Radius problems for some subclasses of analytic functions. Complex Anal. Oper. Theory 2017, 11, 1639-1649. [CrossRef]

10. Kargar, R.; Ebadian, A.; Sokół, J. On Booth lemniscate and starlike functions. Anal. Math. Phys. 2017. [CrossRef]

11. Cho, N.E.; Kumar, S.; Kumar, V.; Ravichandran, V. Differential subordination and radius estimates for starlike functions associated with the Booth lemniscate. Turk. J. Math. 2018, 42, 1380-1399.

12. Srivastava, H.M.; Owa, S. (Eds.) Current Topics in Analytic Function Theory; World Scientific Publishing Co., Inc.: River Edge, NJ, USA, 1992.

13. Dziok, J.; Raina, R.K.; Sokół, J. Certain results for a class of convex functions related to a shell-like curve connected with Fibonacci numbers. Comput. Math. Appl. 2011, 61, 2605-2613. [CrossRef]

14. Dziok, J.; Raina, R.K.; Sokół, J. On a class of starlike functions related to a shell-like curve connected with Fibonacci numbers. Math. Comput. Model. 2013, 57, 1203-1211. [CrossRef]

15. Bell, E.T. The iterated exponential integers. Ann. Math. 1938, 39, 539-557. [CrossRef]

16. Bell, E.T. Exponential polynomials. Ann. Math. 1934, 35, 258-277. [CrossRef]

17. Berndt, B.C. Ramanujan reaches his hand from his grave to snatch your theorems from you. Asia Pac. Math. Newsl. 2011, 1, 8-13.

18. Canfield, E.R. Engel's inequality for Bell numbers. J. Combin. Theory Ser. A 1995, 72, 184-187. [CrossRef]

19. Qi, F. An explicit formula for the Bell numbers in terms of the Lah and Stirling numbers. Mediterr. J. Math. 2016, 13, 2795-2800. [CrossRef]

20. Qi, F. Some inequalities for the Bell numbers. Proc. Indian Acad. Sci. Math. Sci. 2017, 127, 551-564. [CrossRef]

21. Srivastava, H.M.; Manocha, H.L. A Treatise on Generating Functions; Ellis Horwood Series: Mathematics and its Applications; Ellis Horwood Ltd.: Chichester, UK, 1984.

22. Kumar, V.; Cho, N.E.; Ravichandran, V.; Srivastava, H.M. Sharp coefficient bounds for starlike functions associated with the Bell numbers. Math. Slovaca 2019, accepted.

23. Ali, R.M.; Ravichandran, V. Integral operators on Ma-Minda type starlike and convex functions. Math. Comput. Model. 2011, 53, 581-586. [CrossRef]

24. Nunokawa, M.; Obradović, M.; Owa, S. One criterion for univalency. Proc. Am. Math. Soc. 1989, 106, 1035-1037. [CrossRef]

25. Ali, R.M.; Ravichandran, V.; Seenivasagan, N. Sufficient conditions for Janowski starlikeness. Int. J. Math. Math. Sci. 2007, 2007, 62925. [CrossRef]

26. Ali, R.M.; Cho, N.E.; Ravichandran, V.; Kumar, S.S. Differential subordination for functions associated with the lemniscate of Bernoulli. Taiwan. J. Math. 2012, 16, 1017-1026. [CrossRef]

27. Kumar, S.; Ravichandran, V. Subordinations for Functions with Positive Real Part. Complex Anal. Oper. Theory 2018, 12, 1179-1191. [CrossRef]

28. Tuneski, N.; Bulboacă, T.; Jolevska-Tuneska, B. Sharp results on linear combination of simple expressions of analytic functions. Hacet. J. Math. Stat. 2016, 45, 121-128. [CrossRef]

29. Ahuja, O.P.; Kumar, S.; Ravichandran, V. Applications of first order differential subordination for functions with positive real part. Stud. Univ. Babeş-Bolyai Math 2018, 63, 303-311. [CrossRef]

30. Bohra, N.; Kumar, S.; Ravichandran, V. Some Special Differential Subordinations. Hacet. J. Math. Stat. 2018, accepted. [CrossRef]

31. Dziok, J.; Raina, R.K.; Sokół, J. Applications of differential subordinations for norm estimates of an integral operator. Proc. R. Soc. Edinb. Sect. A 2018, 148, 281-291. [CrossRef]

32. Miller, S.S.; Mocanu, P.T. Differential Subordinations: Theory and Applications; Marcel Dekker: New York, NY, USA, 2000; Volume 225.

33. Marjono; Thomas, D.K. Subordination on $\delta$-convex functions in a sector. Honam Math. J. 2001, 23, 41-50.

34. Grunsky, H. Neue abschätzungen zur konformen abbildung ein-und mehrfachzusammenhângender bereiche. Schr. Deutsche Math.-Ver 1934, 43, 140-143. 
35. Sokół, J. Radius problems in the class $\mathcal{S}_{L}^{*}$. Appl. Math. Comput. 2009, 214, 569-573.

36. Ali, R.M.; Jain, N.K.; Ravichandran, V. On the radius constants for classes of analytic functions. Bull. Malays. Math. Sci. Soc. 2013, 36, 23-38.

37. Cho, N.E.; Kumar, S.; Kumar, V.; Ravichandran, V. Convolution and radius problems of analytic functions associated with the Tilted Carathedory functions. Math. Commun. 2019, accepted.

38. Kowalczyk, J.; Les, E.; Sokół, J. Radius problems in a certain subclass of close-to-convex functions. Houst. J. Math. 2014, 40, 1061-1072.

39. Kumar, S.; Ravichandran, V. Functions defined by coefficient inequalities. Malays. J. Math. Sci. 2017, 11, 365-375.

40. Livingston, A.E. On the radius of univalence of certain analytic functions. Proc. Am. Math. Soc. 1966, 17, 352-357. [CrossRef]

41. MacGregor, T.H. The radius of univalence of certain analytic functions. Proc. Am. Math. Soc. 1963, 14, 514-520. [CrossRef]

42. Ravichandran, V.; Rønning, F.R.; Shanmugam, T.N. Radius of convexity and radius of starlikeness for some classes of analytic functions. Complex Var. Theory Appl. 1997, 33, 265-280. [CrossRef]

43. Shah, G.M. On the univalence of some analytic functions. Pac. J. Math. 1972, 43, 239-250. [CrossRef]

(C) 2019 by the authors. Licensee MDPI, Basel, Switzerland. This article is an open access article distributed under the terms and conditions of the Creative Commons Attribution (CC BY) license (http://creativecommons.org/licenses/by/4.0/). 